\title{
Distribution of human papillomavirus genotypes in cervical lesions
}

\author{
LI MA, XIAO CONG, MAI SHI, XIU-HONG WANG, HAI-YAN LIU and MEI-LU BIAN \\ Department of Gynecology and Obstetrics, China-Japan Friendship Hospital, Beijing 100029, P.R. China
}

Received February 26, 2015; Accepted April 6, 2016

DOI: $10.3892 /$ etm.2016.4000

\begin{abstract}
The aims of the present study were to investigate the distribution of human papillomavirus (HPV) genotypes in cervical lesions, and the association between different HPV genotypes and cervical lesions. Between January 2013 and June 2014, the HPV type determinations of nucleic acid by use of fluorescence polymerase chain reaction (PCR) method of 15,192 outpatients in China-Japan Friendship Hospital were performed and the infection status was analyzed. The results showed that: i) 2,366 Cases were HPV positive and 12,826 cases were HPV negative, the overall infection rate was $15.57 \%$ $(2,366 / 15,192)$, in which a single genotype of HPV infection rate was $11.63 \%(1,767 / 15,192)$, and multiple genotypes of HPV infection rate was 3.94\% (599/15,192); ii) HPV16, HPV52 and HPV58 infections were the most common HPV genotypes, the infection rates were $3.95 \%(600 / 15,192), 2.86 \%(435 / 15,192)$ and $2.67 \%(406 / 15,192)$, respectively; and iii) According to the gold standard of histopathological analysis via hematoxylin-eosin staining, HPV16, HPV52 and HPV58 accounted for $58.80 \%$ (154/267) of all CIN2 or above squamous epithelial lesions. Furthermore, three cases with pathological changes of the cervical severe glandular epithelium were all HPV18 infection. The difference was statistically significant $\left(\chi^{2}=60.74, \mathrm{P}<0.001\right)$. Single HPV subtype infection was primarily associated with HPV16, HPV52 and HPV58. In conclusion, HPV type detection had a may be important in screening of cervical lesions as a difference in pathogenic ability was noted among different HPV genotypes. As cervical cancer is an infectious disease, HPV testing may help detect more precancerous lesions, thus reducing the morbidity and mortality of cervical cancer. HPV16, HPV52 and HPV58 were associated with severe cervical squamous epithelial lesions; HPV18 was associated with cervical severe glandular cell pathological changes, although it was not the most common HPV genotype in China. When positive, a clinical cervical examination should be conducted, including colposcopy and biopsy.
\end{abstract}

Correspondence to: Dr Mei-Lu Bian, Department of Gynecology and Obstetrics, China-Japan Friendship Hospital, 2 Yinghua Dongjie, Beijing 100029, P.R. China

E-mail: bianmeilu@hotmail.com

Key words: human papilloma virus, genotype, cervical intraepithelial neoplasia, cervical glandular intraepithelial neoplasia

\section{Introduction}

Cervical cancer is a type of malignant tumor which was the fourth most common cancer amongst women worldwide in 2012, with 528,000 new cases $(1,2)$. Human papillomavirus (HPV) has been considered to be a major cause of cervical cancer and cervical intraepithelial neoplasia, and high-risk (HR) HPV persistent infection significantly correlates with cervical cancer (3-5). HPV genotyping is being increasingly studied, along with the study of the association between various HPV types and cervical cancer. The importance of its clinical application was proposed by the European Research Organization on Genital Infection and Neoplasia (EUROGIN) in 2010 (6). In 2011, the application of HPV genotyping to screening was proposed by the same organization, which can detect more CIN2-3 patients compared with a cytology test (7). Furthermore, Qiao et al (8), Arbyn et al (9) and Liu et al (10) have proposed that high-risk HPV detection may be used for cervical carcinoma screening in China. Significant reform was made in cervical cancer screening in 2015 after the American Society for Colposcopy and Cervical Pathology proposed that HPV genotyping be used as a cervical cancer screening method (11). The possibility of selecting HPV genotyping as the first choice for cervical carcinoma screening was investigated in the present study based on HPV genotyping data collected in the China-Japan Friendship Hospital (Beijing, China).

\section{Materials and methods}

Ethical approval and patient consent. Written informed consent was obtained from all the patients, and this study was approved by the Ethics Committee of the China-Japan Friendship Hospital (Beijing, China).

Study population. A total of 15,192 female outpatients at the China-Japanese Friendship Hospital volunteered to undergo a High-Risk HPV genotyping assay [quantitative polymerase chain reaction (qPCR) method] between January 2013 and June 2014. The average patient age was 32.52 \pm 7.77 years. Participants were sexually active and did not receive surgery for uterine diseases. Participants were recruited while in hospital to undergo physical examination, as well as patients that experienced abnormal leucorrhea, vaginal bleeding during sexual intercourse and abnormal vaginal bleeding.

High-risk HPV genotyping assay (qPCR). Exfoliated cells were collected by swab at cervical foci, placed in sterile glass 
tube and sealed. Genotyping was performed using a Uterine Cervix Cancer of High-risk HPV Genotype Related Real Time PCR Kit (Liferiver Bio-Tech Corp., San Diego, CA, USA), which is able to detect and distinguish 13 HPV genotypes, including HPV16, 18, 31, 33, 35, 39, 45, 51, 52, 56, 58, 59 and 68. A total of 13 pairs of genotype-specific primers and 13 Taqman probes (Liferiver Bio-Tech Corp.) were used for the detection and genotyping of the genomic DNA of $13 \mathrm{HPV}$ genotypes using qPCR.

In brief, template DNA was prepared from cervical swab samples taken from 15,192 female outpatients using Nucleic Acid Extraction Reagent, which is a component of High-risk HPV Genotype Related Real Time PCR Kit. Primers were provided in the kit and the total reaction volume was $40 \mu 1$. Thermal cycling was performed according to the manufacturer's instructions: $94^{\circ} \mathrm{C}$ for $2 \mathrm{~min}$, followed by 40 cycles of $93^{\circ} \mathrm{C}$ for $10 \mathrm{sec}$ and $62^{\circ} \mathrm{C}$ for $31 \mathrm{sec}$. PCR amplification and hybridization were performed using the Roche LightCycler (Roche Diagnostics, Basel, Switzerland). Positive and negative controls were performed, as provided by in the kit.

Fluorescent signals of cycle 6-15 were used for baseline setting, and threshold line was set just over the highest point of amplification curve of blank, in which $\mathrm{H}_{2} \mathrm{O}$ was used as template. Cycle threshold $(\mathrm{Ct})$ value was used as a marker of virus load, $\mathrm{Ct}$ value represents the threshold cycle number of qPCR; higher HPV copy number implies more severe HPV infection in patient and higher risk of developing into cervical cancer. If $\mathrm{Ct}$ value is $<38$ and typical amplification curve is shown, a positive result may be reported; one or more HPV genotypes can be detected, which represent simple and multiple infection respectively. HPV virus load can be divided into seven levels, $10^{2}-10^{7}$ and $>10^{7}$ based on $\mathrm{Ct}$ values: When $\mathrm{Ct}$ value is $<20.0$, HPV viral load is $>10^{7}$ copies $/ 10^{4}$ cells; Ct value is $20.0-23.3$, HPV viral load is $10^{7}$ copies $/ 10^{4}$ cells; $\mathrm{Ct}$ value is 23.4-26.7, HPV viral load is $10^{6}$ copies $/ 10^{4}$ cells; $\mathrm{Ct}$ value is 26.8-30.1, HPV viral load is $10^{5}$ copies $/ 10^{4}$ cells; $\mathrm{Ct}$ value is $30.2-33.5$, HPV viral load is $10^{4}$ copies $/ 10^{4}$ cells; $\mathrm{Ct}$ value is 33.6-36.9, HPV viral load is $10^{3}$ copies $/ 10^{4}$ cells; and $\mathrm{Ct}$ value is $37.0-40.0$, HPV viral load is $10^{2}$ copies $/ 10^{4}$ cells. $\mathrm{Ct}$ value formula of HPV-positive sample: $\mathrm{Ct}=\mathrm{Ct}_{\text {Sample }}-\mathrm{Ct}_{\mathrm{IC}}+28$. If the Ct value of 'Channel CY5' in mixture I [which is able to detect HPV16, 56, 31 and internal control (IC)] is $<32$, which represents IC, and 'Undetermined' or 'No Ct' is shown in 'Column $\mathrm{Ct}$ ' of all channels except IC, the result was considered to be negative. If the $\mathrm{Ct}$ value was between 38.0 and 40.0, the sample was tested repeatedly; if it remained in that interval and a typical S-shaped amplification curve was shown, a result was considered to be positive. If no typical S-shaped curve is observed, a negative result can be reported.

Liquid-based cytology test (LCT). Slides were produced using AutoCyte Prep (TriPath Imaging, Inc., Becton Dickinson, Burlington, NC, USA), and cytology diagnosis was made based on The 2001 Bethesda system (TBS2001) classification system by American Academy of Family Physicians (12). Cytological results were considered to be positive if the clinical significance is over the grade of ASC-H (atypical squamous cells-cannot exclude high-grade squamous intraepithelial lesion). All LCT tests were reviewed and diagnosed by two cytopathologists independently.
Histopathology. Histopathological diagnosis is the gold standard for the diagnosis of cervical lesions. Cervical biopsy samples were extracted from 648 cases in 2,366 HPV-positive patients, including HPV-positive and LCT-positive patients, as well as LCT negative but HPV16-positive patients, in this study by colposcopy. Biopsy tissue was fixed with $10 \%$ formaldehyde, dehydrated, embedded in paraffin and cut into sections (3-4 $\mu \mathrm{m})$ using a microtome. The sections were subjected to hematoxylin-eosin staining (Beyotime Institute of Biotechnology, Inc., Shanghai, China). Sections were observed under a microscope (BX51; Olympus Corp., Tokyo, Japan), reviewed and independently diagnosed by two pathologists. The histopathological diagnosis standard adopted was the World Health Organization classification of tumors pathology (13). Histopathology grades over CIN2 (including CIN2) were considered to be positive, and histopathology grades below CIN1 (including CIN1) were considered to be negative.

Statistical analysis. SPSS 17.0 statistical software (SPSS, Inc., Chicago, IL, USA) was applied for analysis, disaggregated data describing the percentage, rate comparisons using $\chi^{2}$ test, numerical data (mean \pm standard deviation) describing both groups were compared according to homogeneity of variance, nonparametric tests (Mann-Whitney u-test). $\mathrm{P}<0.05$ was considered to indicate a statistically significant difference.

\section{Results}

Age distribution of participants. The HPV infection rate was found to be $15.57 \%(2,366 / 15,192)$ in 15,192 female participants, with an average age of $32.52 \pm 7.77$ years. The average age of the 2,366 HPV-positive patients was 34.53 \pm 9.62 years, and was $32.15 \pm 7.32$ years for the $12,826 \mathrm{HPV}$-negative patients. There was a significant difference $(Z=-9.815$, $\mathrm{P}<0.001)$ between the ages of the HPV-positive and negative participants. Single genotype infection number was 1,767 in HPV-positive patients and the infection rate was $11.63 \%$ $(1,767 / 15,192)$. The multiple infection number was 599 and the infection rate was $3.94 \%(599 / 15,192)$.

Results of high risk HPV genotyping assay ( $q P C R)$. A total of $13 \mathrm{HPV}$ genotypes were detected in this study: HPV16 $(3.95 \%, 600 / 15,192)$, HPV52 $(2.86 \%, 435 / 15,192)$, HPV58 $(2.67 \%, 406 / 15,192)$, HPV39 $(1.98 \%, 301 / 15,192)$, HPV68 $(1.72 \%, 262 / 15,192)$, HPV51 (1.49\%, 226/15,192), HPV56 (1.38\%, 209/15,192), HPV59 (1.36\%, 207/15,192), HPV18 $(1.01 \%, 153 / 15,192)$, HPV31 $(0.68 \%, 104 / 15,192)$, HPV33 $(0.68 \%, 104 / 15,192)$, HPV35 $(0.64 \%, 97 / 15,192)$ and HPV45 $(0.31 \%, 47 / 15,192)$.

Among different age groups, the HPV infection rate varied. From 25 to 29 years old, 838 cases were HPV positive and 5,434 cases were HPV negative, the infection rate was $13.36 \%(838 / 6,272)$. From 30 to 39 years old, 947 cases were HPV positive and 5,571 cases were HPV negative, the infection rate was $14.53 \%(947 / 6,518)$, from 40 to 49 years old, 343 cases were HPV positive and 1,313 cases were HPV negative, the infection rate was $20.71 \%(343 / 1,656)$, from 50 to 59 years old, 187 cases were HPV positive and 431 cases were HPV negative, the infection rate was $30.26 \%(187 / 618)$, 

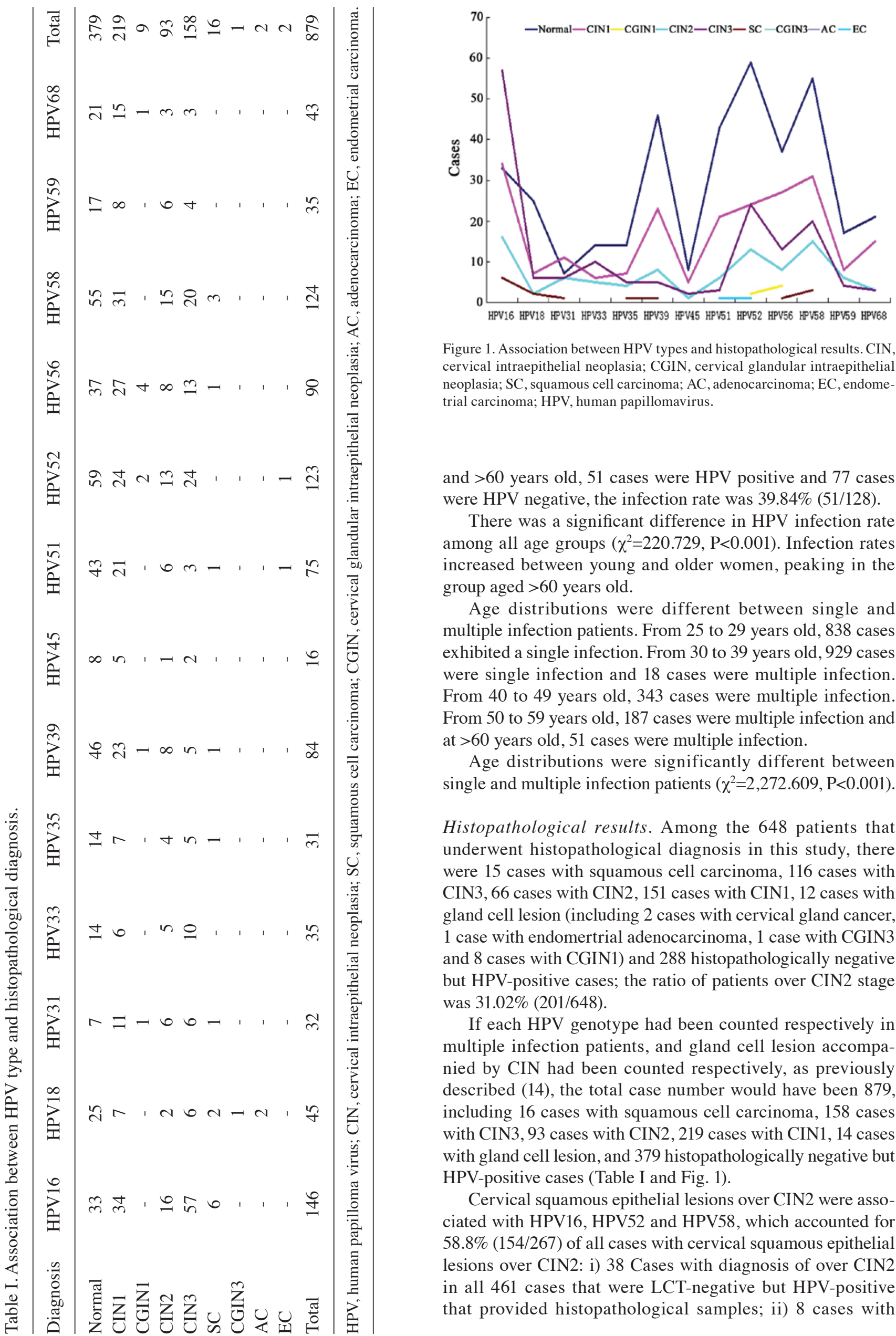

Figure 1. Association between HPV types and histopathological results. CIN cervical intraepithelial neoplasia; CGIN, cervical glandular intraepithelial neoplasia; SC, squamous cell carcinoma; AC, adenocarcinoma; EC, endometrial carcinoma; HPV, human papillomavirus.

and $>60$ years old, 51 cases were HPV positive and 77 cases were HPV negative, the infection rate was 39.84\% (51/128).

There was a significant difference in HPV infection rate among all age groups $\left(\chi^{2}=220.729, \mathrm{P}<0.001\right)$. Infection rates increased between young and older women, peaking in the group aged $>60$ years old.

Age distributions were different between single and multiple infection patients. From 25 to 29 years old, 838 cases exhibited a single infection. From 30 to 39 years old, 929 cases were single infection and 18 cases were multiple infection. From 40 to 49 years old, 343 cases were multiple infection. From 50 to 59 years old, 187 cases were multiple infection and at $>60$ years old, 51 cases were multiple infection.

Age distributions were significantly different between single and multiple infection patients $\left(\chi^{2}=2,272.609, \mathrm{P}<0.001\right)$.

Histopathological results. Among the 648 patients that underwent histopathological diagnosis in this study, there were 15 cases with squamous cell carcinoma, 116 cases with CIN3, 66 cases with CIN2, 151 cases with CIN1, 12 cases with gland cell lesion (including 2 cases with cervical gland cancer, 1 case with endomertrial adenocarcinoma, 1 case with CGIN3 and 8 cases with CGIN1) and 288 histopathologically negative but HPV-positive cases; the ratio of patients over CIN2 stage was $31.02 \%(201 / 648)$.

If each HPV genotype had been counted respectively in multiple infection patients, and gland cell lesion accompanied by CIN had been counted respectively, as previously described (14), the total case number would have been 879 , including 16 cases with squamous cell carcinoma, 158 cases with CIN3, 93 cases with CIN2, 219 cases with CIN1, 14 cases with gland cell lesion, and 379 histopathologically negative but HPV-positive cases (Table I and Fig. 1).

Cervical squamous epithelial lesions over CIN2 were associated with HPV16, HPV52 and HPV58, which accounted for $58.8 \%$ (154/267) of all cases with cervical squamous epithelial lesions over CIN2: i) 38 Cases with diagnosis of over CIN2 in all 461 cases that were LCT-negative but HPV-positive that provided histopathological samples; ii) 8 cases with 

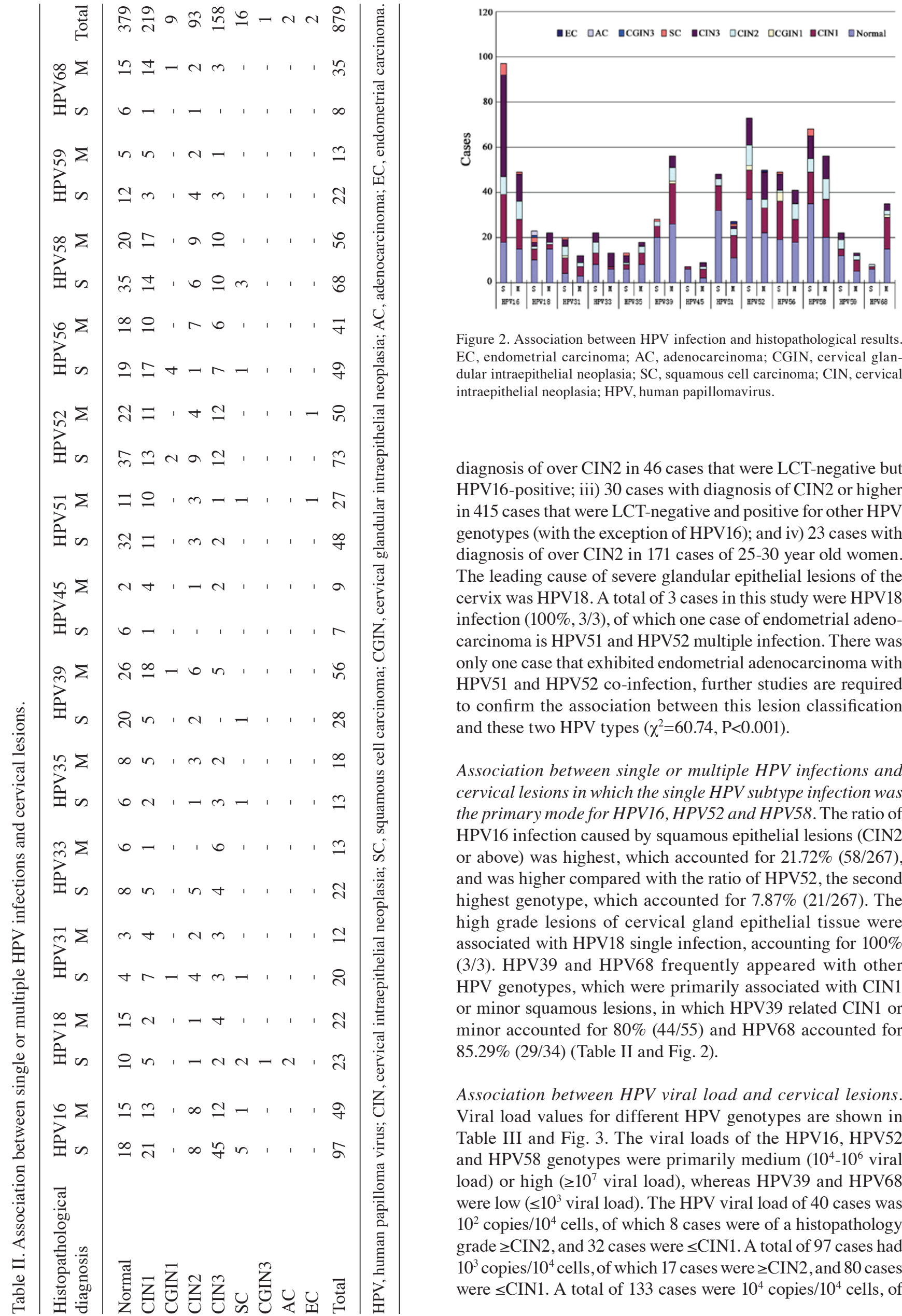

Figure 2. Association between HPV infection and histopathological results. EC, endometrial carcinoma; AC, adenocarcinoma; CGIN, cervical glandular intraepithelial neoplasia; SC, squamous cell carcinoma; CIN, cervical intraepithelial neoplasia; HPV, human papillomavirus.

diagnosis of over CIN2 in 46 cases that were LCT-negative but HPV16-positive; iii) 30 cases with diagnosis of CIN2 or higher in 415 cases that were LCT-negative and positive for other HPV genotypes (with the exception of HPV16); and iv) 23 cases with diagnosis of over CIN2 in 171 cases of 25-30 year old women. The leading cause of severe glandular epithelial lesions of the cervix was HPV18. A total of 3 cases in this study were HPV18 infection $(100 \%, 3 / 3)$, of which one case of endometrial adenocarcinoma is HPV51 and HPV52 multiple infection. There was only one case that exhibited endometrial adenocarcinoma with HPV51 and HPV52 co-infection, further studies are required to confirm the association between this lesion classification and these two HPV types $\left(\chi^{2}=60.74, P<0.001\right)$.

Association between single or multiple HPV infections and cervical lesions in which the single HPV subtype infection was the primary mode for HPV16, HPV52 and HPV58. The ratio of HPV16 infection caused by squamous epithelial lesions (CIN2 or above) was highest, which accounted for $21.72 \%$ (58/267), and was higher compared with the ratio of HPV52, the second highest genotype, which accounted for 7.87\% (21/267). The high grade lesions of cervical gland epithelial tissue were associated with HPV18 single infection, accounting for $100 \%$ (3/3). HPV39 and HPV68 frequently appeared with other HPV genotypes, which were primarily associated with CIN1 or minor squamous lesions, in which HPV39 related CIN1 or minor accounted for $80 \%(44 / 55)$ and HPV68 accounted for 85.29\% (29/34) (Table II and Fig. 2).

Association between HPV viral load and cervical lesions. Viral load values for different HPV genotypes are shown in Table III and Fig. 3. The viral loads of the HPV16, HPV52 and HPV58 genotypes were primarily medium $\left(10^{4}-10^{6}\right.$ viral load) or high ( $\geq 10^{7}$ viral load), whereas HPV39 and HPV68 were low ( $\leq 10^{3}$ viral load). The HPV viral load of 40 cases was $10^{2}$ copies $/ 10^{4}$ cells, of which 8 cases were of a histopathology grade $\geq$ CIN2, and 32 cases were $\leq$ CIN1. A total of 97 cases had $10^{3}$ copies $/ 10^{4}$ cells, of which 17 cases were $\geq$ CIN 2 , and 80 cases were $\leq$ CIN1. A total of 133 cases were $10^{4}$ copies $/ 10^{4}$ cells, of 


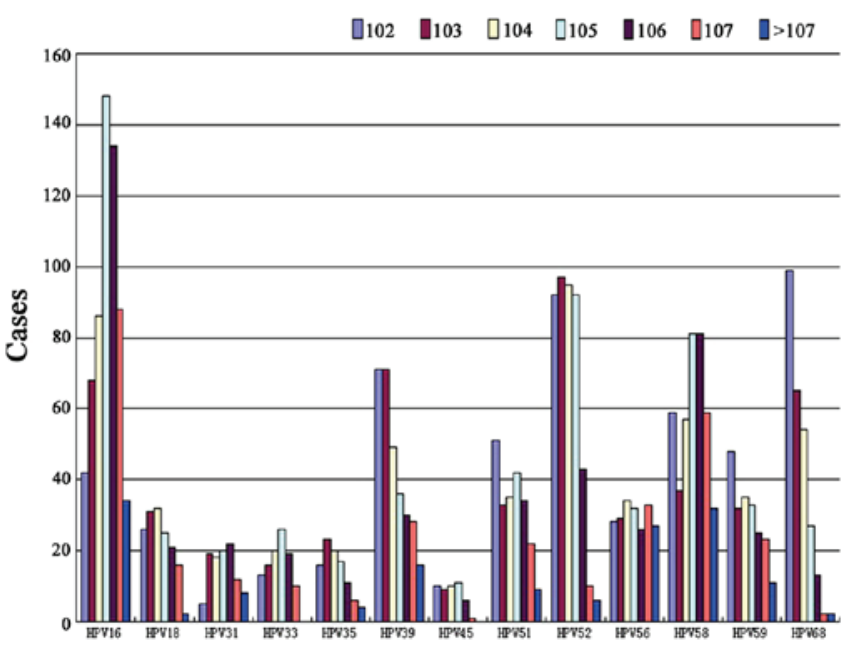

Figure 3. Viral load of different HPV types. (HPV, human papilloma virus).

which 34 cases were $\geq$ CIN2, and 99 cases were $\leq$ CIN1. A total of 163 cases were $10^{5}$ copies $/ 10^{4}$ cells, of which 43 cases were $\geq \mathrm{CIN} 2$, and 120 cases were $\leq \mathrm{CIN} 1$. A total of 172 cases were $10^{6}$ copies $/ 10^{4}$ cells, of which 73 cases were $\geq$ CIN 2 and 99 cases were $\leq$ CIN1. A total of 144 cases were $10^{7}$ copies $/ 10^{4}$ cells, of which 50 cases were $\geq \mathrm{CIN} 2$, and 94 cases were $\leq \mathrm{CIN} 1$. A total of 130 cases were $>10^{7}$ copies $/ 10^{4}$ cells, of which 47 cases were $\geq$ CIN2 and 83 cases were $\leq$ CIN1.

The percentages of the viral loads were as follows: $4.55 \%$ (40/879) for $10^{2} / 10^{4}$ cells; $11.04 \%$ (97/879) for $10^{3} / 10^{4}$ cells; $15.13 \%(133 / 879)$ for $10^{4} / 10^{4}$ cells; $18.54 \%(163 / 879)$ for $10^{5} / 10^{4}$ cells; $19.57 \%(172 / 879)$ for $10^{6} / 10^{4}$ cells; $16.38 \%$ (144/879) for $10^{7} / 10^{4}$ cells; and $14.79 \%$ (130/879) for $>10^{7} / 10^{4}$ cells.

The viral loads of squamous cell carcinoma, adenocarcinoma and endometrial carcinoma patients were $>10^{4} / 10^{4}$ cells in all patients (16/16 for squamous cell carcinoma, $2 / 2$ for adenocarcinoma and $2 / 2$ for endometrial carcinoma). The ratio of the viral load of $>10^{4} / 10^{4}$ cells accounted for $87.10 \%$ (81/93) in CIN2 and 91.77\% (145/158) in CIN3, which suggested that the cervical lesion type was associated with viral load. The percentage of cervical lesions of CIN2 or above was $18.25 \%$ $(25 / 137)$ in patients with viral load of $\leq 10^{3} / 10^{4}$ cells and $33.29 \%$ (247/742) for $>10^{4} / 10^{4}$ cells viral load patients. There was a significant difference $\left(\chi^{2}=27.06, P<0.001\right)$ between these two groups.

\section{Discussion}

The incidence and mortality rates of cervical carcinoma in China accounted for $\sim 1 / 3$ of the global total in 2002 (15). Early prevention, early detection and early treatment are the key to reduce morbidity and mortality. The development of cervical carcinoma is a gradual process, and may require $\geq 10$ years to develop into invasive cervical carcinoma from dysplasia (16). Persistent HPV infection is the most important risk factor for cervical carcinoma, and high-risk HPV and cervical carcinoma are closely correlated $(17,18)$.

According to a worldwide retrospective cross-sectional study conducted in 2010, the top 8 invasive cervical carcinoma HPV genotypes are HPV16, HPV18, HPV31, HPV33, HPV35, 
HPV45, HPV52 and HPV58 in Europe, America, Africa, Asia and Pacific areas (3). The overall HPV infection rate was $15.57 \%(2,366 / 15,192)$ in the present study, and the most common genotypes are HPV16 $(3.95 \%, 600 / 15,192)$, HPV52 $(2.86 \% 435 / 15,192)$ and HPV58 $(2.67 \% 406 / 15,192)$. In previous studies by Chen et al (19), Shi et al (20) and Li et al (21), the HPV infection rates were $19.7 \%$ in Xinjiang (China), $32 \%$ in Changsha (China) and 9.9\% in Beijing (China), respectively, and the most common genotypes were HPV16/HPV58/HPV18, HPV52/HPV16/HPV58 and HPV16/HPV58/HPV33, respectively.

It remains to be further verified by considerably large sample numbers whether cervical carcinoma-related HPV genotype prevalence in China is different from that in the rest of the world.

Previous studies $(22,23)$ have demonstrated that there was a difference in pathogenic ability among different HPV genotypes, and that infection with high-risk HPV genotypes in particular may pose a health risk to female patients. High-risk HPV genotype infections were the risk factors of the lesions diagnosed CIN 2 or higher. When HPV16/18-positive, colposcopy should be performed regardless of the LCT results. In 2007, the International Agency for Research on Cancer proposed that the HPV test may be used as a primary screening method for cervical carcinoma (22). In 2012, the guidelines of the American Congress of Obstetricians and Gynecologists and the American Society for Colposcopy and Cervical Pathology stated that women infected with HPV16 and HPV18 should be examined by colposcopy, even if cytologically negative (23). In the present study, there were $21.05 \%(8 / 38)$ of HPV16-positive cases in patients with LCT negative results and CIN2 or above in histopathologic diagnosis. Therefore, direct HPV genotyping should be considered, if the required laboratory facilities are available. For example, the Cobas HPV genotyping assay (Roche Diagnostics, Risch-Rotkreuz, Switzerland), which was approved by the American Food and Drug Administration in April 2014, may be used as a method for cervical disease screening. Recent updates to cervical lesion screening guidelines indicate the increasing importance of HPV genotyping $(22,24,25)$.

In a previous study, HPV16 and HPV18 were considered as the most two common cervical carcinoma related high-risk genotypes (26). In the present study, cervical lesions of CIN2 or above accounted for $31.02 \%$ (201/648) of $648 \mathrm{HPV}$ positive cases with histopathological data. The infection rate of HPV16 was the highest, $29.59 \%(79 / 267)$ in patients with cervical squamous intraepithelial lesions of CIN2 or above and higher compared with that of HPV58, the second highest genotype accounting for $14.23 \%$ (38/267). HPV18 infection rate was $5.12 \%(45 / 879)$ overall, but $3.75 \%(10 / 267)$ in patients with cervical squamous intraepithelial lesions of CIN2 or above. However, HPV18 was important in severe cervical glandular epithelial lesions, 1 case of CGIN3 and 2 cases of cervical adenocarcinoma were all associated with HPV18 single infection in the present study. Cervical glandular epithelial lesions are easily neglected, as their locations are frequently too deep to detect and there have been few studies investigating cervical glandular epithelial lesions. Cervical canal should be examined to reduce misdiagnosis of cervical glandular epithelial lesions, if HPV18 infection is detected. In addition, higher viral load indicates higher possibility of severe cervical lesions. In the present study, the incidence of severe cervical lesions in the group with the viral load $>10^{4}$ was higher compared with the $<10^{4}$ group.

There are three strategies of cervical carcinoma screening for healthy women in the USA $(22,24,25,27)$ : i) Cytological screening alone for women over 21-year-old; ii) HPV screening was the prime choice for women $>25$-year-old; and iii) cytological and HPV joint screening for women $>30$-year-old. The screening strategies above are for healthy women, but this study is opportunistic screening. In the present study, participants between 25 and 30-years-old with cervical lesions of CIN2 or above accounted for $0.37 \%(23 / 6,272)$ in that age group, whereas the incidence was $2.00 \%(178 / 8,920)$ in the group $>30$-years-old. According to the results above, the incidence still existed in the group of 25-30-year-old women, although the incidence was low. Therefore, young women should meet at least one of following requirements before selecting HPV genotyping as the first screen method: i) 25-30 Years old with previous abnormal LCT history; and ii) good financial conditions. In general, HPV genotyping of cervical carcinoma screening is first choice for women $>30$-years-old.

In conclusion, HPV genotyping indicates that patients with HPV16, HPV52 and HPV58 infections may be particularly susceptible to high-grade cervical intraepithelial lesions, and the endocervix should be examined for patients with HPV18 infection. Thereby, the detection rate of severe cervical squamous intraepithelial lesions and severe glandular intraepithelial lesions may be improved and the incidence of cervical carcinoma may be reduced. HPV genotyping is feasible and economical as the first choice of opportunistic screening in tertiary hospitals.

\section{Acknowledgements}

The authors thank the clinical doctors of the Department of Gynecology and Obstetrics, China-Japan Friendship Hospital for their kind assistance in the collection of data, and thank the pathologists of the Department of Pathology (China-Japan Friendship Hospital) for their assistance in the diagnosis.

\section{References}

1. Ferlay J, Soerjomataram I, Ervik M, Dikshit R, Eser S, Mathers C, Rebelo M, Parkin DM, Forman D and Bray F: GLOBOCAN 2012 v1.0, Cancer Incidence and Mortality Worldwide: IARC CancerBase No. 11 (Internet). International Agency for Research on Cancer, Lyon, France, 2013. http://globocan.iarc.fr. Accessed December 12, 2013

2. Bray F, Ren JS, Masuyer E and Ferlay J: Global estimates of cancer prevalence for 27 sites in the adult population in 2008. Int J Cancer 132: 1133-1145, 2013.

3. Ronco G, Giorgi-Rossi P, Carozzi F, Confortini M, Dalla Palma P, Del Mistro A, Ghiringhello B, Girlando S, Gillio-Tos A, De Marco L, et al: New technologies for cervical Cancer screening (NTCC) working group: Efficacy of human papillomavirus testing for the detection of invasive cervical cancer and cervical intraepithelial neoplasia: A randomised controlled trial. Lancet Oncol 11: 249-257, 2010.

4. Cox JT, Castle PE, Behrens CM, Sharma A, Wright TC Jr and Cuzick J; Athena HPV Study Group: Comparison of cervical cancer screening strategies incorporating different combinations of cytology, HPV testing, and genotyping for HPV 16/18: Results from the ATHENA HPV study. Am J Obstet Gynecol 208: 184.e1-184.e11, 2013. 
5. Castle PE, Stoler MH, Wright TC Jr, Sharma A, Wright TL and Behrens CM: Performance of carcinogenic human papillomavirus (HPV) testing and HPV16 or HPV18 genotyping for cervical cancer screening of women aged 25 years and older: A subanalysis of the ATHENA study. Lancet Oncol 12: 880-890, 2011.

6. Franceschi S, Denny L, Irwin KL, Jeronimo J, Lopalco PL, Monsonego J, Peto J, Ronco G, Sasieni P and Wheeler CM: EUROGIN 2010 roadmap on cervical cancer prevention. Int J Cancer 128: 2765-2774, 2011.

7. Li N, Franceschi S, Howell-Jones R, Snijders PJ and Clifford GM: Human papillomavirus type distribution in 30,848 invasive cervical cancers worldwide: Variation by geographical region, histological type and year of publication. Int J Cancer 128: 927-935, 2011.

8. Qiao YL, Sellors JW, Eder PS, Bao YP, Lim JM, Zhao FH, Weigl B, Zhang WH, Peck RB, Li L, et al: A new HPV-DNA test for cervical cancer screening in developing regions: A cross-sectional study of clinical accuracy in rural China. Lancet Oncol 9: 929-936, 2008

9. Arbyn M, Wallker A and Meijer CJ: HPV-based cervical cancer screening in China. Lancet Oncol 11: 1112-1113, 2010.

10. Liu Q, Wang B, Kong Y and Cheng KK: China's primary health-care reform. Lancet 377: 2064-2066, 2011.

11. Huh WK, Ault KA, Chelmow D, Davey DD, Goulart RA, Garcia FA, Kinney WK, Massad LS, Mayeaux EJ, Saslow D, et al: Use of primary high-risk human papillomavirus testing for cervical cancer screening: Interim clinical guideline. Obstet Gynecol 125: 330-337, 2015.

12. Apgar BS, Zoschnick L and Wright TC Jr: The 2001 Bethesda System terminology. Am Fam Physician 68: 1992-1998, 2003.

13. Wells M: Epithelial tumors of the uterine cervix. In: Pathology and Genetics of Tumors of the Breast and Female Genital Organs. Tavassoli FA and Devilee P (eds). IARC Press, Lyon, pp262-276, 2003.

14. Bian ML, Cheng JY, Ma L, Cong X, Liu J, Chen Y and Chen X: Evaluation of the detection of 14 high-risk human papillomaviruses with HPV 16 and HPV 18 genotyping for cervical cancer screening. Exp Ther Med 6: 1332-1336, 2013.

15. Lang JH: Global challenges and opportunities in cervical cancer prevention. Zhong Guo Fu Chan Ke Lin Chuang Za Zhi 37: 129-131, 2002 (In Chinese).

16. Wang Q: The analysis of 132 cases of cervical carcinoma Zhong Guo Xian Dai Yi Sheng 48: 109-132, 2010 (In Chinese).

17. Cai YF and Zhu GZ: The role high- risk human papillomavirus DNA test and cytological detection in cervical cancer screening. Zhong Guo Fu You Bao Jian 21: 3434, 2006 (In Chinese).
18. Pan LY: HPV infection and preinvasive disease of cervix. Ji Chu Yi Xue Yu Lin Chuang 12: 1296-1301, 2006 (In Chinese).

19. Chen ZF, Ma He-FZ, Ding Y and Ma XH: The analysis of HPV infection status in women of Han, Uyghur and Kazakhs in Xinjiang, China. Zhong Guo Shi Yong Fu Ke Yu Chan Ke Za Zhi 8: 602-605, 2011 (In Chinese).

20. Shi LC, He J, He L and Wang W: The research of HPV infection and genotype distribution of 13542 women in Changshang. Jilin Med J 34: 3384-3386, 2013 (In Chinese).

21. Li C, Wu M, Wang J, Zhang S, Zhu L, Pan J and Zhang W: A population-based study on the risks of cervical lesion and human papillomavirus infection among women in Beijing, People's Republic of China. Cancer Epidemiol Biomarkers Prev 19: 2655-2664, 2010.

22. Wright TC Jr, Massad LS, Dunton CJ, Spitzer M, Wilkinson EJ and Solomon D; 2006 American Society for Colposcopy and Cervical Pathology-sponsored Consensus Conference: 2006 consensus guidelines for the management of women with abnormal cervical cancer screening tests. Am J Obstet Gynecol 197: 346-355, 2007.

23. Committee on Practice Bulletins-Gynecology: ACOG practice bulletin number 131: Screening for cervical cancer. Obstet Gynecol 120: 1222-1238, 2012.

24. Massad LS, Einstein MH, Huh WK, Katki HA, Kinney WK, Schiffman M, Solomon D, Wentzensen N and Lawson HW; 2012 ASCCP Consensus Guidelines Conference: 2012 updated consensus guidelines for the management of abnormal cervical cancer screening tests and cancer precursors. Obstet Gynecol 121: 829-846, 2013.

25. Saslow D, Solomon D, Lawson HW, Killackey M, Kulasingam SL, Cain J, Garcia FA, Moriarty AT, Waxman AG, Wilbur DC, et al: American Cancer Society, American Society for Colposcopy and Cervical Pathology and American Society for Clinical Pathology screening guidelines for the prevention and early detection of cervical cancer. Am J Clin Pathol 137: 516-542, 2012

26. Josefsson AM, Magnusson PK, Ylitalo N, Sørensen P, Qwarforth-Tubbin P, Andersen PK, Melbye M, Adami HO and Gyllensten UB: Viral load of human papilloma virus 16 as a determinant for development of cervical carcinoma in situ: A nested case-control study. Lancet 355: 2189-2193, 2000.

27. Massad LS, Einstein MH, Huh WK, Katki HA, Kinney WK, Schiffman M, Solomon D, Wentzensen N and Lawson HW; 2012 ASCCP Consensus Guidelines Conference: 2012 updated consensus guidelines for the management of abnormal cervical cancer screening tests and cancer precursors. J Low Genit Tract Dis 17 (5 Suppl 1): S1-S27, 2013. 